\title{
Multiregional Tumor Drug-Uptake Imaging by PET and Microvascular Morphology in End-Stage Diffuse Intrinsic Pontine Glioma
}

\author{
Sophie E.M. Veldhuijzen van Zanten*1,2 ${ }^{*}$ A. Charlotte P. Sewing*1,2 Arthur van Lingen $^{3}$, Otto S. Hoekstra ${ }^{3}$, \\ Pieter Wesseling ${ }^{2,4,5}$, Michaël H. Meel ${ }^{1,2}$, Dannis G. van Vuurden ${ }^{1,2}$, Gertjan J.L. Kaspers ${ }^{1,6}$, Esther Hulleman ${ }^{1,2}$, \\ and Marianna Bugiani ${ }^{4}$ \\ ${ }^{I}$ Division of Oncology/Haematology, Department of Pediatrics, VUmc, Amsterdam, The Netherlands; ${ }^{2}$ Neuro-Oncology Research \\ Group, Cancer Center Amsterdam, VUmc, Amsterdam, The Netherlands; ${ }^{3}$ Department of Radiology and Nuclear Medicine, VUmc, \\ Amsterdam, The Netherlands; ${ }^{4}$ Department of Pathology, VUmc, Amsterdam, The Netherlands; ${ }^{5}$ Department of Pathology, Princess \\ Máxima Center for Pediatric Oncology, Utrecht, The Netherlands; and ${ }^{6}$ Department of Pediatrics, Princess Máxima Center for \\ Pediatric Oncology, Utrecht, The Netherlands
}

See an invited perspective on this article on page 610 .

Inadequate tumor uptake of the vascular endothelial growth factor antibody bevacizumab could explain lack of effect in diffuse intrinsic pontine glioma. Methods: By combining data from a PET imaging study using ${ }^{89} \mathrm{Zr}$-labeled bevacizumab and an autopsy study, a 1-on-1 analysis of multiregional in vivo and ex vivo ${ }^{89} \mathrm{Zr}$-bevacizumab uptake, tumor histology, and vascular morphology in a diffuse intrinsic pontine glioma patient was performed. Results: In vivo ${ }^{89} \mathrm{Zr}$-bevacizumab measurements showed heterogeneity between lesions. Additional ex vivo measurements and immunohistochemistry of cervicomedullary metastasis samples showed uptake to be highest in the area with marked microvascular proliferation. In the primary pontine tumor, all samples showed similar vascular morphology. Other histologic features were similar between the samples studied. Conclusion: In vivo ${ }^{89} \mathrm{Zr}-$ bevacizumab PET serves to identify heterogeneous uptake between tumor lesions, whereas subcentimeter intralesional heterogeneity could be identified only by ex vivo measurements. ${ }^{89} \mathrm{Zr}$-bevacizumab uptake is enhanced by vascular proliferation, although our results suggest it is not the only determinant of intralesional uptake heterogeneity.

Key Words: brain stem neoplasm; PET; ${ }^{89} \mathrm{Zr}$-bevacizumab; microvascular morphology; VEGF

J Nucl Med 2018; 59:612-615

DOI: 10.2967/jnumed.117.197897

$\mathbf{E}$ nd-stage diffuse intrinsic pontine glioma (DIPG) shows glioblastoma histology (1) and overexpression of proangiogenic factors, including vascular endothelial growth factor (VEGF) $(1,2)$. However, whether DIPG patients benefit from anti-VEGF treatment, such

Received Jun. 27, 2017; revision accepted Aug. 7, 2017.

For correspondence or reprints contact: Sophie E.M. Veldhuijzen van Zanten, VU University Medical Center, Division of Oncology/Hematology, Department of Pediatrics, De Boelelaan 1118, 1081HZ Amsterdam, The Netherlands.

E-mail: s.veldhuijzen@vumc.nl

${ }^{*}$ Contributed equally to this work.

Published online Aug. 17, 2017.

COPYRIGHT (C 2018 by the Society of Nuclear Medicine and Molecular Imaging. as the monoclonal antibody bevacizumab, is still unclear (3-5). So far, the results of trials with bevacizumab have been disappointing $(6,7)$. A recent drug imaging study assessing tumor uptake of ${ }^{89} \mathrm{Zr}$ labeled bevacizumab in DIPG showed inter- and intrapatient heterogeneity, but factors determining uptake are currently unknown (8). Therefore, in this study we performed a 1-on-1 analysis of multiregional in vivo and ex vivo ${ }^{89} \mathrm{Zr}$-bevacizumab uptake, tumor histology, and vascular morphology in a DIPG patient.

\section{MATERIALS AND METHODS}

A 12-y-old girl presented with paralysis of the left abducens nerve. Brain MR imaging revealed a T1-weighted hypointense, T2-weighted hyperintense lesion infiltrating $50 \%$ of the pons (9), suggestive of DIPG. Biopsy demonstrated high-grade diffuse glioma features, and Sanger sequencing of $H 3 F 3 A$ and $H I S T 1 H 3 B$ revealed a wild-type status (10). Whole-exome sequencing showed no mutations in HIST1H3C or HIST2H3C either. A diagnosis of histone H3 wild-type DIPG was made (11). The patient was enrolled in phase A of the VUmc-01 DIPG study (Dutch Trial Register: NTR2391) and treated with radiotherapy (54 Gy [30 treatments of 1.8-Gy each]) and weekly gemcitabine $\left(175 \mathrm{mg} / \mathrm{m}^{2} / \mathrm{wk}\right)$ for $6 \mathrm{wk}$. Progression-free survival was $3.8 \mathrm{mo}$. After disease progression, the patient participated in a molecular imaging study (NTR3518), including an immuno-PET scan at $145 \mathrm{~h}$ after injection of a $0.1 \mathrm{mg} / \mathrm{kg}$ dose of bevacizumab labeled with ${ }^{89} \mathrm{Zr}(0.9 \mathrm{MBq} / \mathrm{kg})$, as previously described (8). Four days afterward, the patient died (death was unrelated to study participation) and participated in an autopsy study. All 3 studies were approved by the Institutional Review Board of VUmc and performed in accordance with the Declaration of Helsinki. For each study, both parents gave written informed consent $(8,12,13)$.

\section{In Vivo ${ }^{89} \mathrm{Zr}$-Bevacizumab Uptake Measurements}

In vivo ${ }^{89} \mathrm{Zr}$-bevacizumab uptake was quantified as decay-corrected maximum activity concentration (in $\mathrm{Bq} / \mathrm{mL}$ ) in manually delineated volumes of interest, that is, tumor areas with visually enhanced uptake. Activity concentrations were also converted into SUVs, that is, decaycorrected maximum activity concentration normalized to injected dose per body weight.

\section{Ex Vivo ${ }^{89} \mathrm{Zr}$-Bevacizumab Uptake Measurements}

Brain autopsy was performed $2 \mathrm{~h}$ after death. Multiple $0.5-\mathrm{cm}^{3}$ tumor and control samples (macroscopically nonaffected brain, cerebrospinal fluid, and blood) were obtained for ex vivo ${ }^{89} \mathrm{Zr}$-radioactivity 
measurements and histologic analysis. Ex vivo radioactivity concentrations were measured with a $\gamma$-well counter (1480 Wizard; Wallac). The in vivo PET scanner (Ingenuity TF-128 PET/CT scanner; Philips Healthcare) was cross-calibrated with the well counter $(14,15)$. Ex vivo ${ }^{89} \mathrm{Zr}$-radioactivity concentrations were also quantified as corrected maximum activity concentration and converted into SUV.

\section{Immunohistochemistry}

Four-micrometer-thick formalin-fixed, paraffin-embedded tissue sections were stained with hematoxylin and eosin and immunostained as previously validated and described $(16,17)$ against glial fibrillary acidic protein (1:400; Dako); MIB-1 (1:40; Dako); glucose transporter 1 (1:200; Thermo Scientific), smooth muscle actin (1:200; Dako), collagen-IV (1:50; Dako), CD34 (1:50; Dako), and VEGF (1:50; Pharmagen). Specific stainings were chosen to determine differences in proliferation grade in the vascular endothelial cells (MIB-1) of the tumor; to highlight vascular morphology by identifying endothelial cells (CD34), vascular smooth muscle cells, activated pericytes (smooth muscle actin), and the vascular basal membrane (collagenIV); to visualize tumor morphology and resident astrocytes (glial fibrillary acidic protein); to identify blood-brain barrier integrity and areas of increased hypoxia (glucose transporter 1); and to determine VEGF expression. Immunopositivity was detected with 3,3'diaminobenzidine. Omitting primary antibodies yielded no significant staining. Sections were photographed using a Leica DM6000B microscope.

\section{RESULTS}

\section{In Vivo ${ }^{89} \mathrm{Zr}$-Bevacizumab Uptake}

The T1-weighted postgadolinium MR images obtained $8 \mathrm{~d}$ before death showed a $3.6 \times 3.3 \mathrm{~cm}$ primary pontine tumor, with strong contrast enhancement but no apparent necrosis. Furthermore, extensive meningeal and subependymal metastases in the right ventricular trigone (VTM) and cervicomedullary junction (CMM) (Fig. 1), as well as in the right frontal lobe, left Sylvian fissure, left lateral ventricle posterior horn, and along the spinal cord, were observed (Supplemental Figs. 1A-1F; supplemental materials are available at http://jnm.snmjournals.org). At $145 \mathrm{~h}$, the most intense ${ }^{89} \mathrm{Zr}$-bevacizumab uptake positivity was seen in the CMM (corrected maximum activity concentration, 7,800 Bq/mL; SUV, 9.9; volume, $2.0 \mathrm{~mL}$ ), followed by the primary pontine tumor $(5,607 \mathrm{~Bq} / \mathrm{mL}$; SUV, 7.1 ; volume, $13.1 \mathrm{~mL})$ and the VTM $(2,537 \mathrm{~Bq} / \mathrm{mL}$; SUV, 3.2; volume, $1.2 \mathrm{~mL}$ ).

\section{Ex Vivo ${ }^{89}$ Zr-Bevacizumab Uptake}

Ex vivo radioactivity measurements at $242 \mathrm{~h}$ were obtained for multiple tumor samples (Fig. 2A), including the VTM, primary tumor, CMM, and a small 0.02-g fragment of dural metastasis that was previously undetected by MRI and PET (Supplemental Fig. 2 ), and macroscopically normal areas of the pons, cerebellum, and dura. Tracer uptake was highest in the dural metastasis $(22,652$ $\mathrm{Bq} / \mathrm{mL}$; SUV, 28.7) and CMM samples, with a considerable difference between the cranial $(27,152 \mathrm{~Bq} / \mathrm{mL}$; SUV, 34.4) and caudal $(16,184 \mathrm{~Bq} / \mathrm{mL}$; SUV, 20.5) parts. Differences in uptake were also observed between the cranial $(2,949 \mathrm{~Bq} / \mathrm{mL}$; SUV, 3.7) and caudal $(11,343 \mathrm{~Bq} / \mathrm{mL}$; SUV, 14.4) parts of the primary tumor. Radioactivity was low in normal tissue samples $(<1,844 \mathrm{~Bq} / \mathrm{mL}$; SUV, $<2.3)$, cerebrospinal fluid (62 Bq/mL; SUV, 0.1), and blood (716 Bq/mL; SUV, 0.9).

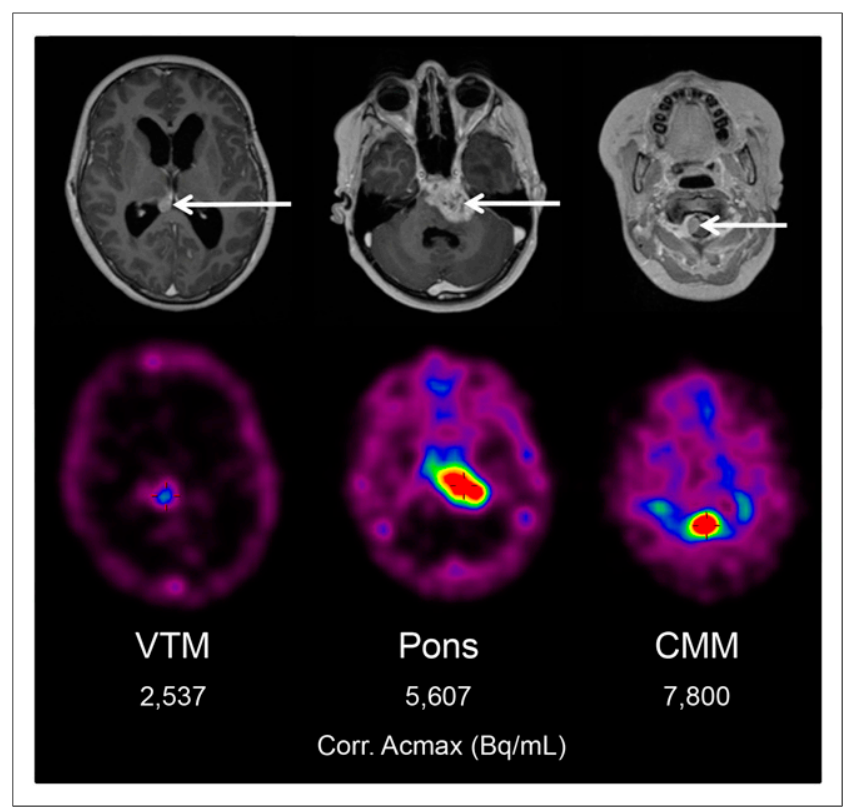

FIGURE 1. Gadolinium-enhanced T1-weighted MR images obtained 8 $\mathrm{d}$ before death, and corresponding PET slices obtained $4 \mathrm{~d}$ before death (145 $\mathrm{h}$ after ${ }^{89} \mathrm{Zr}$-bevacizumab injection), showing primary pontine tumor and metastases in VTM and CMM. Arrows represent areas of disease. Corr. Acmax $=$ corrected maximum activity concentration.

\section{Correlation Between In Vivo and Ex Vivo 89Zr-Bevacizumab Uptake}

Figure $2 \mathrm{~B}$ shows the ratio between the in vivo and ex vivo ${ }^{89} \mathrm{Zr}-$ bevacizumab uptake of the primary tumor, VTM, and CMM.

\section{Correlation Between Ex Vivo ${ }^{89} \mathrm{Zr}$-Bevacizumab Uptake and Histology}

After ex vivo radioactivity measurements, multiple samples from the primary tumor and CMM were investigated with histochemistry and immunohistochemistry to explore differences in histology as a possible explanation for tracer uptake heterogeneity. Hematoxylin and eosin staining showed a diffuse growth pattern, high cellularity, and variable cytonuclear atypia (Figs. 3A and 3B) in all tumor samples and confirmed the absence of tumor in macroscopically normal tissue. Necrosis and florid glomeruloid microvascular proliferation were present only in the cranial CMM (with the highest ex vivo radioactivity) (Fig. 3A). In this sample, MIB-1 staining was positive in up to $50 \%$ of tumor cells and in numerous endothelial cells in the microvascular walls (Fig. 3B). In the remaining samples, microvascular proliferation was less pronounced: here, 30\% (in both primary tumor samples) to $50 \%$ (in the caudal CMM sample) of tumor cell nuclei were MIB-1-positive, whereas endothelial cells were MIB-1-negative (Fig. 3B). Glial fibrillary acidic protein was expressed in tumor cells in the primary tumor and CMM, as well as in residual nonneoplastic astrocytes (Fig. 3C). Staining of CD34 showed partial loss of microvascular immunoreactivity in the caudal part of the primary tumor. In the other samples, CD34 immunopositivity was maintained (Fig. 3D). Immunoreactivity for glucose transporter 1 was similar in all samples (Fig. 3E). Staining of collagen-IV and smooth muscle actin highlighted the vascular basal lamina and tunica media, respectively, in all samples without obvious differences (Fig. 3E). Staining of VEGF was similar among all samples (Fig. 3F). 


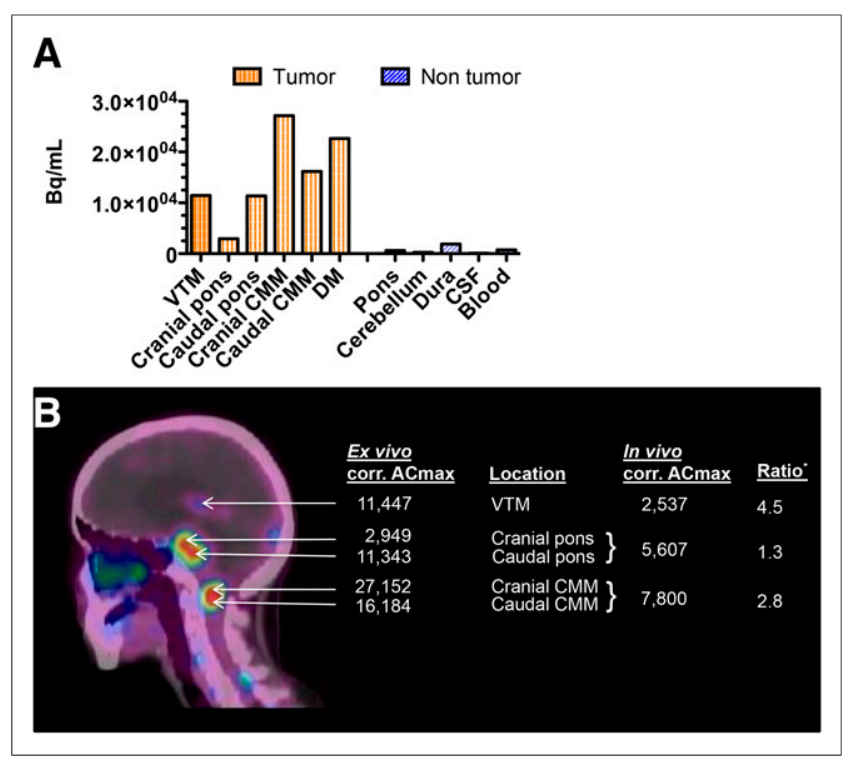

FIGURE 2. (A). Ex vivo ${ }^{89} \mathrm{Zr}$-bevacizumab uptake ( $242 \mathrm{~h}$ after injection) in tumor samples and macroscopically normal tissue samples, cerebrospinal fluid, and blood. (B) Correlation with in vivo ${ }^{89} \mathrm{Zr}$-bevacizumab uptake (arrows, $145 \mathrm{~h}$ after injection). *Numerator for ex vivo uptake of primary tumor and CMM is average of cranial and caudal corrected maximum activity concentration (corr. ACmax). DM = dural metastasis; $\mathrm{CSF}=$ cerebrospinal fluid.

\section{DISCUSSION}

This study directly correlated multiregional ${ }^{89} \mathrm{Zr}$-bevacizumab uptake with tumor histology and vascular morphology in a patient with histone H3 wild-type DIPG (12). Observed differences between in vivo and ex vivo radioactivity measurements reflected tracer pharmacokinetics (e.g., ongoing tracer clearance from blood over time) in combination with spatially and/or temporally heterogeneously increasing ${ }^{89} \mathrm{Zr}$-bevacizumab tissue deposition. Moreover, in vivo PET underestimates the true activity in small lesions (in this case, the CMM and VTM) because of partial-volume effects. Because of this effect (14), the actual radioactivity concentration as assessed by imaging at $145 \mathrm{~h}$ after ${ }^{89} \mathrm{Zr}$-bevacizumab injection was likely underestimated by approximately $70 \%$. Finally, as opposed to ex vivo measurements, at $145 \mathrm{~h}$ intravascular ${ }^{89} \mathrm{Zr}$-bevacizumab still contributes to the in vivo PET tumor signal (blood pool SUV, 4) (8). Thus, whereas in vivo PET serves to identify whole-body tracer distribution and heterogeneity between lesions, the subcentimeter intralesional heterogeneity of tracer distribution can be defined only with ex vivo measurements.

Interestingly, hematoxylin and eosin and MIB-1 staining showed prominent microvascular proliferation, with higher ${ }^{89} \mathrm{Zr}-$ bevacizumab uptake, in the cranial part of the CMM versus the caudal part, with lower uptake. No other histologic differences were found between the two samples, such as pattern of tumor growth, extent of necrosis, or difference in immunoreactivity for endothelial markers. In the two samples from the pons, intralesional uptake heterogeneity was also observed, although here both areas showed similar vascular morphology, tumor growth, and extent of necrosis. These findings suggest that vascular proliferation is an important, yet not the only, determinant of intralesional heterogeneity in ${ }^{89} \mathrm{Zr}$-bevacizumab uptake.
The presence of microvascular proliferation and disorganized vessels (18) in World Health Organization grade IV glioma is considered a consequence of hypoxia-induced overexpression of proangiogenic factors, including $\operatorname{VEGF}(1,2)$. In the cranial CMM sample, glomeruloid microvascular structures with increased endothelial cell turnover (as determined by MIB-1immunoreactivity) were prominent. On this basis, VEGF was expected to be overexpressed. VEGF immunoreactivity, however, showed mostly aspecific staining and did not differ between samples. This may be explained by the fact that the very short half-life of the VEGF protein (15-20 $\min$ (19)) was incompatible with its immunohistochemical detection $2 \mathrm{~h}$ after death. Glucose transporter 1 staining was similar in all samples analyzed, suggesting a partially intact blood-brain barrier. However, our histologic analysis did not provide definitive clues to determine the role of blood-brain barrier integrity in ${ }^{89} \mathrm{Zr}$-bevacizumab uptake in this case.

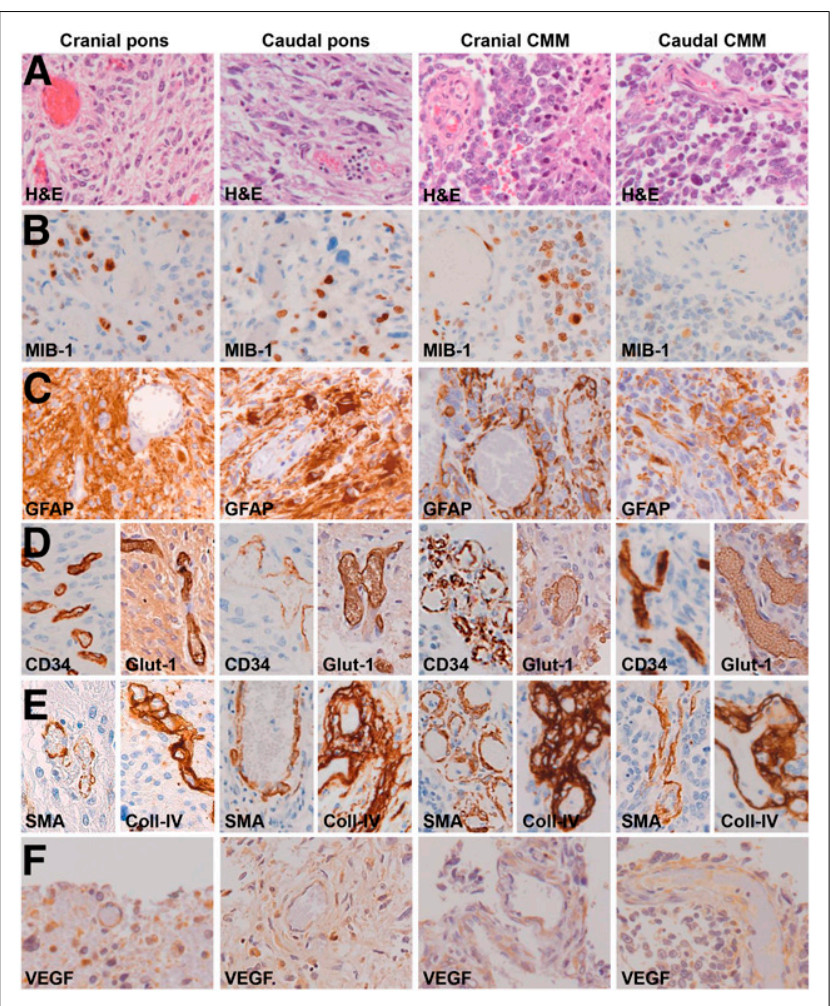

FIGURE 3. Histology and immunohistochemistry of samples from cranial and caudal parts of primary pontine tumor and CMM, respectively: hematoxylin and eosin (A); MIB-1 (for detection of proliferating cells) (B); astrocyte marker glial fibrillary acidic protein (C); CD34 (endothelial cell marker) and Glut-1 (glucose transporter expressed by brain endothelial cells as part of blood-brain barrier, and often deregulated in areas with hypoxia) (D); smooth muscle actin (marker of vascular smooth muscle cells and activated pericytes) and collagen-IV (component of vascular basal membrane) (E); and VEGF staining (F). All images are $200 \times$ original magnification. Diffuse growth pattern is seen in all samples. Extensive necrosis and glomerular microvascular proliferation is seen with multiple MIB-1-positive (endothelial) cells in microvascular walls in caudal CMM. Partial loss of CD-34 is seen in caudal pons. Similar staining against glial fibrillary acidic protein, glucose transporter 1 , and VEGF is seen in all samples. All vessels and microvessels are highlighted in stain against collagen-IV and smooth muscle actin. 


\section{CONCLUSION}

Although concerning a single case, we conclude that in vivo PET is capable of detecting heterogeneity in ${ }^{89} \mathrm{Zr}$-bevacizumab uptake between lesions, which correlates well with ex vivo measurements. However, PET cannot detect subcentimeter intralesional uptake heterogeneity. Furthermore, our results suggest that targeting of ${ }^{89} \mathrm{Zr}$-bevacizumab is enhanced in areas with vascular proliferation. However, because significant inter- and intralesional heterogeneity was also observed in areas that did not show differences in vascular proliferation, other factors present in the DIPG microenvironment likely also play a role. Because the DIPG microenvironment is heterogeneous and dynamic (3), patients are likely to fail treatment if timing and patient selection are not optimized. The results of this study underline the potential of immuno-PET studies, especially when combined with biopsy or autopsy studies, in the quest for optimal selection and timing of treatment schedules.

\section{DISCLOSURE}

This research was funded by the Semmy Foundation (Stichting Semmy). No other potential conflict of interest relevant to this article was reported.

\section{ACKNOWLEDGMENT}

We thank Marc Huisman (Department of Radiology and Nuclear Medicine, VUmc) for assistance with ex vivo and in vivo calculations.

\section{REFERENCES}

1. Buczkowicz P, Bartels U, Bouffet E, Becher O, Hawkins C. Histopathological spectrum of paediatric diffuse intrinsic pontine glioma: diagnostic and therapeutic implications. Acta Neuropathol (Berl). 2014;128:573-581.

2. Puget S, Philippe C, Bax DA, et al. Mesenchymal transition and PDGFRA amplification/mutation are key distinct oncogenic events in pediatric diffuse intrinsic pontine gliomas. PLoS One. 2012;7:e30313.

3. Ballester LY, Wang Z, Shandilya S, et al. Morphologic characteristics and immunohistochemical profile of diffuse intrinsic pontine gliomas. Am J Surg Pathol. 2013;37:1357-1364.

4. Gururangan S, Chi SN, Young Poussaint T, et al. Lack of efficacy of bevacizumab plus irinotecan in children with recurrent malignant glioma and diffuse brainstem glioma: a Pediatric Brain Tumor Consortium study. J Clin Oncol. 2010;28:3069-3075.

5. Jansen MHA, Lagerweij T, Sewing ACP, et al. Bevacizumab targeting diffuse intrinsic pontine glioma: results of ${ }^{89} \mathrm{Zr}$-bevacizumab PET imaging in brain tumor models. Mol Cancer Ther. 2016;15:2166-2174.

6. Parekh C, Jubran R, Erdreich-Epstein A, et al. Treatment of children with recurrent high grade gliomas with a bevacizumab containing regimen. J Neurooncol. 2011;103:673-680.

7. Zaky W, Wellner M, Brown RJ, Blüml S, Finlay JL, Dhall G. Treatment of children with diffuse intrinsic pontine gliomas with chemoradiotherapy followed by a combination of temozolomide, irinotecan, and bevacizumab. Pediatr Hematol Oncol. 2013;30:623-632.

8. Jansen MH, Veldhuijzen van Zanten SE, Van Vuurden DG, et al. Molecular drug imaging: ${ }^{89} \mathrm{Zr}$-bevacizumab PET in children with diffuse intrinsic pontine glioma. J Nucl Med. 2017;58:711-716.

9. Barkovich AJ, Krischer J, Kun LE, et al. Brain stem gliomas: a classification system based on magnetic resonance imaging. Pediatr Neurosurg. 1990-1991;16: 73-83.

10. Wu G, Broniscer A, McEachron TA, et al. Somatic histone H3 alterations in pediatric diffuse intrinsic pontine gliomas and non-brainstem glioblastomas. Nat Genet. 2012;44:251-253.

11. Louis DN, Perry A, Reifenberger G, et al. The 2016 World Health Organization classification of tumors of the central nervous system: a summary. Acta Neuropathol (Berl). 2016;131:803-820.

12. Caretti V, Jansen MHA, van Vuurden DG, et al. Implementation of a multiinstitutional diffuse intrinsic pontine glioma autopsy protocol and characterization of a primary cell culture. Neuropathol Appl Neurobiol. 2013;39: 426-436.

13. Bugiani M, Veldhuijzen van Zanten SEM, Caretti V, et al. Deceptive morphologic and epigenetic heterogeneity in diffuse intrinsic pontine glioma. Oncotarget. 2017;8:60447-60452.

14. Makris NE, Boellaard R, Visser EP, et al. Multicenter harmonization of ${ }^{89} \mathrm{Zr}$ PET/CT performance. J Nucl Med. 2014;55:264-267.

15. Greuter HNJM, Boellaard R, van Lingen A, Franssen EJF, Lammertsma AA. Measurement of ${ }^{18} \mathrm{~F}-\mathrm{FDG}$ concentrations in blood samples: comparison of direct calibration and standard solution methods. J Nucl Med Technol. 2003;31:206209.

16. Kevelam SH, Bugiani M, Salomons GS, et al. Exome sequencing reveals mutated SLC19A3 in patients with an early-infantile, lethal encephalopathy. Brain. 2013;136:1534-1543.

17. Navis AC, Hamans BC, Claes A, et al. Effects of targeting the VEGF and PDGF pathways in diffuse orthotopic glioma models. J Pathol. 2011;223:626-634.

18. Wagemakers M. Angiogenesis and Angiopoietins in Human Gliomas [thesis]. Groningen, The Netherlands: University of Groningen; 2015.

19. Levy NS, Chung S, Furneaux H, Levy AP. Hypoxic stabilization of vascular endothelial growth factor mRNA by the RNA-binding protein HuR. J Biol Chem. 1998;273:6417-6423. 